\title{
Topical steroids in the treatment of central and paracentral corneal ulcers
}

\author{
T R Carmichael, Y Gelfand, N H Welsh
}

\begin{abstract}
During an 18-month period a prospective randomised trial was conducted on $\mathbf{4 0}$ selected patients with bacterial corneal ulcers. Two groups were compared: one was treated with antibiotic only and the other with antibiotic plus steroid. Complications were similar in the two groups. No delay in healing rate of the ulcers was seen with the use of topical steroid. Two methods of assessing visual improvement are discussed, but it was not possible to demonstrate a difference in visual outcome between the two groups.
\end{abstract}

The treatment of central (primarily infectious) corneal ulcers with topical steroids has remained a controversial subject since the introduction of steroids in the $1950 \mathrm{ss}^{1}$ The use of steroids is a two-edged sword; they may protect the cornea from host inflammatory response but at the risk of enhancing the infection. There has been no controlled clinical trial to resolve the issue. This is due mainly to a lack of suitable clinical material combined with a large number of variables, such as type of ulcer, isolates, and others.

Experimental data, mainly in rabbits, suggest no adverse effects so long as an appropriate antibiotic is given concurrently with steroids. This has been shown for Pseudomonas aeruginosa ${ }^{23}$ and Staphylococcus aureus ${ }^{4}$ infections. However, Smolin $e t a l^{5}$ suggested that wound healing may be delayed and there may be prolonged replication of organisms in pseudomonas infections. Fungal infections seem adversely affected by the use of steroid because the available antifungals achieve only fungistatic levels in the cornea. Clinical studies ${ }^{78}$ have supported these experimental findings.

The present study compares the results of treating one group of corneal ulcer patients receiving antibiotic only with another group receiving antibiotic plus steroid.

\section{Patients and methods}

The patients included in the study had central or paracentral bacterial corneal ulcers severe enough to warrant hospital admission. Patients were excluded if they had fungal isolates, perforated ulcers or descemetocoeles, underlying viral corneal conditions, atopic ulcers, no light perception on admission, or were less than 13 years of age. On admission the patients were informed of the treatment to be given and consented to it.

Treatment consisted of Kefzol eye drops (cefazolin, fortified, $32 \mathrm{~g} / \mathrm{l}$ ) and gentamicin eye drops (fortified, $14 \mathrm{~g} / \mathrm{l}$ ) hourly, Atropine eyedrops $1 \%$ twice daily, chloromycetin eye oint- ment at night (2200) and twice daily multivitamin tablets (Multivites). In addition subconjunctival cefazolin (125 mg) and gentamicin (20 $\mathrm{mg}$ ) were given on admission, the following morning, and one or two further injections as indicated by the severity of the infection. The subconjunctival antibiotics were injected separately into a bleb of lignocaine. No oral or intravenous antibiotics were used. Once accepted into the study, the patients were randomised and both groups received the same treatment for 24 hours. On the day after admission, if the steroid group was considered to be the same or improved, Maxidex eye drops (dexamethasone $0 \cdot 1 \%$ ) four times a day were added to their treatment and continued until healing was complete, with a minimum of two weeks. If there was frank deterioration the steroid was withheld for 24 hours and the case reassessed and so on.

\section{ASSESSMENT OF RESULTS}

Recording sheets were used to document the initial appearance of the ulcer and its size and depth by means of a drawing. The appearance and best corrected visual acuity were noted on the same sheet during the follow-up period. Follow-up was continued until the final best corrected visual acuity was determined (minimum of four weeks). All assessments were done by two doctors, which was the most objective method of assessment in view of practical limitations (rotation of doctors through teaching hospitals). In measuring visual acuity the same method with the same Snellen chart was used by both doctors.

To analyse statistically the visual acuity data visual acuity was converted to a number (see Table 1). This meant that no light perception was assigned the number 1 and $6 / 6$ was assigned the number 15 . Visions between these values were: (a) Snellen chart values; $(b)$ counting fingers

Table 1 Visual acuities (follow-up at 2 months)

\begin{tabular}{|c|c|c|c|c|c|c|c|}
\hline \multicolumn{4}{|c|}{ Steroid group } & \multicolumn{4}{|c|}{ Non-steroid group } \\
\hline Initial & N.eq & Final & N.eq & Initial & N.eq & Final & N.eq \\
\hline $0 / 80$ & 11 & $20 / 20$ & 15 & CF $3 \mathrm{~m}$ & 6 & $20 / 40$ & 13 \\
\hline $\mathrm{HN}$ & 3 & $20 / 200$ & 9 & $\mathrm{HM}$ & 3 & NLP & 1 \\
\hline $20 / 40$ & 13 & $20 / 30$ & 14 & $\mathrm{CF} 1 \mathrm{~m}$ & 4 & CF $5 \mathrm{~m}$ & 8 \\
\hline CF $4 \mathrm{~m}$ & 7 & $20 / 200$ & 9 & $20 / 80$ & 11 & $20 / 40$ & 13 \\
\hline LP & 2 & LP & 2 & $\mathrm{HM}$ & 3 & CF $3 \mathrm{~m}$ & 6 \\
\hline HM & 3 & CF lm & 4 & $20 / 120$ & 10 & $20 / 20$ & 15 \\
\hline CF $4 \mathrm{~m}$ & 7 & $20 / 40$ & 13 & $\mathrm{HM}$ & 3 & $20 / 200$ & 9 \\
\hline $\mathrm{HM}$ & 3 & $\mathrm{CF} 1 \mathrm{~m}$ & 4 & HM & 3 & $\mathrm{HM}$ & 3 \\
\hline CF $5 \mathrm{~m}$ & 8 & $20 / 80$ & 11 & $\mathrm{HM}$ & 3 & $\mathrm{HM}$ & 3 \\
\hline $20 / 200$ & 9 & $20 / 120$ & 10 & $20 / 20$ & 15 & $20 / 20$ & 15 \\
\hline LP & 2 & $20 / 120$ & 10 & $\mathrm{CF} 1 \mathrm{~m}$ & 4 & $20 / 80$ & 11 \\
\hline CF $2 \mathrm{~m}$ & 5 & $20 / 40$ & 13 & HM & 3 & CF $5 \mathrm{~m}$ & 8 \\
\hline $\begin{array}{l}C F 1 \mathrm{~m} \\
\mathrm{CF} 1 \mathrm{~m}\end{array}$ & $\begin{array}{l}4 \\
4\end{array}$ & $20 / 200$ & 9 & $20 / 20$ & 15 & $20 / 20$ & 15 \\
\hline $\begin{array}{l}\text { CF 1 m } \\
20 / 200\end{array}$ & $\begin{array}{l}4 \\
9\end{array}$ & $\begin{array}{l}20 / 120 \\
20 / 60\end{array}$ & $\begin{array}{l}10 \\
12\end{array}$ & & & & \\
\hline
\end{tabular}

N.eq is numerical equivalent.

Ophthalmology,
University of the
Witwatersrand,
Johannesburg, Republic
of South Africa
T R Carmichael
Y Gelfand
N H Welsh
Correspondence to:
Dr T R Carmichael, PO Box
2681, Alberton 1450, Republic
of South Africa.
Accepted for publication
11 April 1990




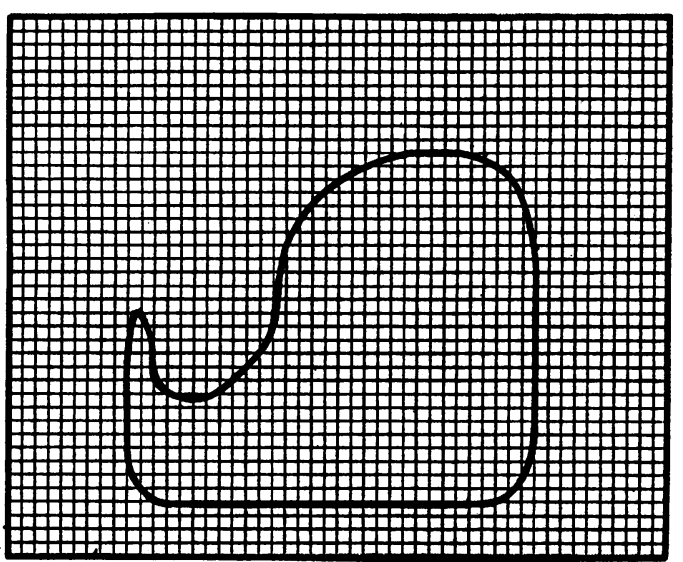

Figure 1 Scale drawing of corneal ulcer on graph paper.

(CF) from 1 to 5 metres; (c) hand movements (HM); (d) light perception (LP); (e) no light perception (NLP).

For purpose of comparison between the groups the transition, for example, between $6 / 24$ and $6 / 36$ is regarded as being the same as a deterioration from CF $1 \mathrm{~m}$ to CF $2 \mathrm{~m}$.

Results of treatment were assessed in terms of: (1) complications; (2) healing rate $\left(\mathrm{mm}^{2}\right.$ of ulcer per day); (3) visual acuity.

\section{BACTERIOLOGY}

Corneal specimens were taken with a platinum spatula at the slit-lamp. These were inoculated on to blood agar and into serum broth and brainheart infusion broth. Two glass slides were prepared, one for Gram stain and one for a special stain if required. Both base and edges of the ulcer were sampled after necrotic debris was removed. Specimens were processed by the Bacteriology Department of Baragawanath Hospital by one technician.

\section{AREA OF ULCERS}

Each ulcer was drawn to scale on to $1 \mathrm{~mm}$ ruled graph paper and the squares counted. This gave an area for each ulcer, on admission, based on the dimensions measured at the slit-lamp (Fig 1). The size of the ulcer in $\mathrm{mm}^{2}$ was used in dividing the ulcers into small (under $3 \mathrm{~mm}^{2}$ ), medium (3-10 $\mathrm{mm}^{2}$ ) and large (over $10 \mathrm{~mm}^{2}$ ) ulcers, to compare the groups further. The area of ulcer healed per day was then calculated to compare the rate of epithelialisation.

\section{Results}

There were 21 eyes in the steroid group and 19 in the non-steroid group. One patient had bilateral corneal ulcers and they were randomised separately. The groups were statistically comparable in terms of number of eyes in each group, age, and duration of inpatient treatment. There

Table 2 Comparison of groups

\begin{tabular}{lll}
\hline & Steroid & Non-steroid \\
\hline No of eyes & 21 & 19 \\
Mean age (years) & $51 \cdot 6$ & $51 \cdot 4$ \\
Percentage males & 90 & 76 \\
Inpatient days (mean) & 18 & 20 \\
\hline
\end{tabular}

Table 3 Comparison of ulcers (numbers)

\begin{tabular}{llcc}
\hline & & Steroid & Non-steroid \\
\hline Site: & Central & 7 & 9 \\
Depth: & Paracentral & 14 & 10 \\
& Outer third & 10 & 8 \\
& Middle third & 5 & 9 \\
& Inner third & 6 & 2 \\
Size: & Small & 9 & 8 \\
& Medium & 8 & 5 \\
& Large & 4 & 6 \\
Hypopyon present & 16 & 12 \\
Positive culture & 14 & 14 \\
\hline
\end{tabular}

were significantly more males in the steroid group $\left(\chi^{2}, \mathrm{p}<0.01\right)$ (Table 2$)$. The patients' ages ranged from 19 to 81 years. There was no significant difference between the ulcers in the two groups with respect to depth, size, positive cultures, or presence of hypopyon (Table 3). Table 4 shows that the corneal isolates obtained were similar in both groups and comparable to the isolates previously obtained in this population. ${ }^{9}$ Complications (Table 5) were similar in the two groups.

Within the steroid group one case showed descemetocoele formation the morning after admission, and, according to the protocol, steroids were not started. There was corneal perforation the following day, and, as the anterior chamber did not reform after pressure padding, a penetrating keratoplasty was performed. Corneal thinning with early descemetocoele formation made it necessary to discontinue steroids after 12 days in one case. The ulcer subsequently healed uneventfully.

One patient with a pneumococcal infection

Table 4 Corneal cultures

\begin{tabular}{lcc}
\hline & Steroid & Non-steroid \\
\hline $\begin{array}{l}\text { Str pneumoniae } \\
\text { Str agalacticae }\end{array}$ & 3 & 4 \\
Str viridans & 3 & 1 \\
$\begin{array}{l}\text { Staph aureus } \\
\text { Staph epidermidis }\end{array}$ & 2 & 1 \\
Bacillus sp & & 3 \\
Haemophilus influenzae & & 1 \\
Pseudomonas paucimobilis & & 1 \\
Proteus mirabilis & & 1 \\
Morganella morganii & 1 & 1 \\
Serratia sp & 1 & \\
Acinetobacter antitratus & 5 & 1 \\
Corynebacteria sp & 7 & 5 \\
No growth & 22 & 21 \\
Total & & \\
\hline
\end{tabular}

Mixed culture in steroid group: Acinetobacter anitratus with Staph epidermidis.

Mixed cultures in non-steroid group: Str pneumoniae with $H$ influenzae Pr mirabilis with Staph epidermidis.

Table 5 Complications

\begin{tabular}{lll}
\hline & Steroid & Non-steroid \\
\hline Perforation & 1 & 1 \\
Corneal thinning & 1 & 1 \\
Uncontrolled infection & 0 & 2 \\
Recurrence of infection & 1 & 2 \\
Persistent epithelial defect & 4 & 3 \\
Epithelial breakdown & 1 & 1 \\
Total & 8 & 10 \\
\hline
\end{tabular}

Persistent epithelial defect is failure of healing of the ulcer within 21 days.

Recurrence of infection is recurrence of abscess/hypopyon occurring within one week of discharge where the infective element was previously considered cleared.

Epithelial breakdown is breakdown of epithelium after initial healing.

One patient in the steroid group had both persistent epithelial defect and recurrent infection. 
showed a persistent epithelial defect (epithelial defect over 21 days) and then presented five days after discharge with a recurrence of corneal abscess and hypopyon. This patient appears under both headings in Table 5. Persistent epithelial defects were seen in four cases. Two of these were large ulcers and healed slowly, and two had severe climatic droplet keratopathy (CDK). In one case the ulcer healed in five days, but the epithelium broke down and rehealing took a further seven days (12 days for final healing). Steroid therapy was discontinued after 12 days in the case of corneal thinning and not given in the case of corneal perforation. All other cases received full courses of topical steroid.

Within the non-steroid group a pneumococcal ulcer perforated after three days but responded to conservative treatment (pressure padding) and healed without surgery. One patient showed corneal thinning and descemetocoele formation but responded to pressure padding and healed uneventfully. Uncontrolled infection occurred in two eyes; one was a Staph aureus infection; in the other case the eye was eviscerated but only Staph epidermidis was isolated. Infection recurred in a patient whose hypopyon had cleared and who was discharged 11 days after admission with a remaining epithelial defect. This patient presented five days later with fresh corneal infiltrate and hypopyon and was readmitted. In the other patient with recurrent infection the ulcer healed within 21 days but the corneal abscess recurred and he was readmitted to hospital.

There were three cases of persistent epithelial defect, two of which were large ulcers and one showed severe CDK. The patient with epithelial breakdown also had CDK, but the ulcerated area rehealed in under 21 days.

Healing rates were calculated for 15 ulcers in the steroid group and 11 in the non-steroid group. Patients were excluded from this analysis if they showed persistent epithelial defects, as some never healed completely, and others took two or three months, which would have distorted the analysis. Patients were also excluded if they required therapy other than that in the protocol for example, pressure padding for perforations or corneal thinning - as this might influence the rate of healing. The two patients with uncontrolled infection did not heal and were excluded from the analysis.

The only patients with complications (Table 5) that were included in the healing rate analysis were those with epithelial breakdown and one with recurrent infections in the non-steroid group. These three patients all showed healing within 21 days. This meant that six patients from the steroid group and eight from the non-steroid group were excluded. There was no significant difference when the two groups were compared by Student's $t$ test. The mean healing rate for the steroid group was $0.36 \mathrm{~mm}^{2} /$ day and for the non steroid group was $0.30 \mathrm{~mm}^{2} /$ day. When the visual acuities between the two groups are compared by the numerical system in Table $I$, no difference could be shown. A statistically significant improvement in both groups followed treatment, as might be expected. The steroid group had an average pretreatment vision of 6 and an

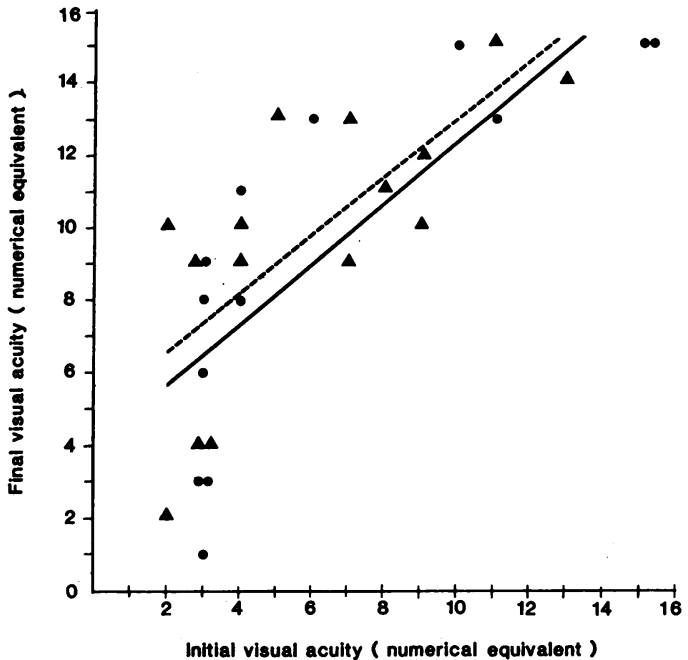

Figure 2 Scattergram with linear regression lines comparing visual improvement with and without steroids.

Non-steroid - A Steroid -

average post-treatment vision of 9.7 ( $p<0.001$, paired analysis by $t$ test). The non-steroid group had an average 6.4 pretreatment and 9.2 posttreatment ( $\mathrm{p}<0.01$ paired analysis by $t$ test). Visual acuities were also compared by plotting scattergrams for pre- and post-treatment visual acuities for each group and fitting linear regression lines. There was again no discernible difference between the two groups, though the improvement in the steroid group was slightly greater (Fig 2). The coefficient of determination $\left(R^{2}\right)$ was $53 \%$ for the steroid group and $64 \%$ for the non-steroid group.

\section{Discussion}

Inflammation and subsequent scarring of the cornea may compromise vision. When designing a treatment regimen some consideration must be given to including an anti-inflammatory agent if this can be done safely. Evidence against the use of steroids include possible detrimental effect should fungal ${ }^{6}$ or viral ${ }^{10}$ infection be present; recurrence of pseudomonas infection ${ }^{11}{ }^{12}$; or prolonged replication of organisms and delayed wound healing. ${ }^{5}$ Supporting the use of steroid is the experimental evidence that pseudomonas ${ }^{23}$ and Staph aureus ${ }^{4}$ keratitis are not adversely affected by steroid use so long as the appropriate antibiotic is used. There are also clinical reports of the use of steroid in bacterial keratitis. ${ }^{78}$ Wilson ${ }^{13}$ mentions that topical steroids were used 'in some but not all' of the 200 bacterial corneal ulcers he treated over a 10-year period.

In the present study the two groups were similar in most respects, and the preponderance of males in the steroid group should not cause undue bias. The ulcers within each group were also comparable, though the isolates differed somewhat. Obviously the larger the groups, the greater the chance that one can collect totally comparable groups. Benefits or adverse effects of using steroids were assessed in the most objective ways possible under the circumstances. Complications within the two groups were remarkably similar, and the feared perforations and corneal thinning in the steroid treated group did not materialise. 
Severe infections may recur, and this was seen with a pneumococcal ulcer in the steroid group. Recurrence of infection was otherwise associated with conditions causing persistent epithelial defects. As previously reported, ${ }^{14}$ the severe nodular form of climatic droplet keratopathy is a common predisposing factor for corneal ulceration in this population. The plaques flake off leaving epithelial defects which are slow to heal and become reinfected. Patterson and Jones ${ }^{10}$ reported using area of ulcers as a means of assessing the negative effects of topical steroids on dendritic keratitis. If accurate measurements are taken at the slit-lamp and the ulcers are carefully drawn to scale on to graph paper, group comparison is possible and any inaccuracies should tend to cancel out. During the present study the method was found to be consistent, and it provides another means of assessment.

No delay in healing rates was seen with the dosage of steroid used. If corneal inflammation and scarring can be reduced by the concurrent use of topical steroids, it should be possible to show an improvement in visual acuity. Two methods of comparing the visual acuities were used, but neither showed a significant difference between the groups. When visions were converted to a numerical quantity, a statistically significant improvement in vision was seen in both groups following treatment, but the steroid group did not do better. Scattergrams with linear regression lines showed very similar visual improvements in the two groups. The correlation coefficients were not particularly high, and this should be taken into account when interpreting the scattergram. In the present study reduction in neovascularisation and conjunctival inflammation and speed of resolution of corneal infiltrate and hypopyon were not measured.

\section{CONCLUSIONS}

No adverse effects were encountered with topical steroids in the dosage shown above. To demonstrate benefits from steroids a larger study would be needed and perhaps some refinements in assessment techniques. It is suggested that steroid treatment should be delayed for 24 hours to exclude rapidly deteriorating infections, allow appropriate selection of antibiotic, and exclude as many fungal infections as possible. It would seem prudent to reduce or discontinue steroid treatment if healing is delayed. Special care should be exercised when treating Streptococcus pneumoniae and Pseudomonas aeruginosa infections, as they can become active again after an initial positive response to antibiotics. A large prospective study is needed, perhaps on a multicentric basis, to collect enough similar cases for meaningful analysis. Different steroid doses need to be assessed to optimise our approach. Complications within a steroid treated group of patients must be accepted, as the present study clearly confirms that complications will occur with and without steroid treatment.

1 Gordon DM, Mclean JM, Koteen H. Present status of corticotropin (ACTH), cortisone, and hydrocortisone in ophthalmology. Brf Ophthalmol 1953; 37: 85-98.

2 Suie T, Taylor FW. The effect of cortisone on experimental pseudomonas corneal ulcers. Arch Ophthalmol 1956; 56: 53-6.

3 Davis SD, Sarff LD, Hyndiuk RA. Corticosteroid in experimentally induced pseudomonas keratitis. Arch Ophthalmol 1978; 96: 126-8.

4 Leibowitz HM, Kupferman A. Topically administered corticosteroids: effect on antibiotic-treated bacterial corticosteroids: effect on antibiotic-treated

5 Smolin G, Okumoto M, Leong-Sit L. Combined gentamicintobramycin-corticosteroid treatment. ii. Effect on gentamicin-resistant pseudomonas keratitits. Arch Ophthalmol 1980; 98: 473-4.

6 O'Day DM, Ray WA, Robinson R, Head WS. Efficacy of antifungal agents in the cornea. ii. Influence of corticoanteroids. Invest Ophthalmol Vis Sci 1984; 25: 331-5.

7 Aronson SB, Moore TE Jr. Corticosteroid therapy in central stromal keratitis. Am $\mathcal{F}$ Ophthalmol 1969; 67: 873-96.

8 Aronson SB, Moore TE Jr. Corticosteroids in infectious corneal disease. In: Leopold IH, ed. Symposium on ocular therapy. St Louis: Mosby, 1972; 5: 77-89.

9 Carmichael TR, Wolpert M, Koornhof HJ. Corneal ulceration at an urban African hospital. $\mathrm{Br} \mathcal{F}$ Ophthalmol 1985; 69: $920-6$.

10 Patterson A, Jones B. The management of ocular herpes. Trans Ophthalmol Soc UK 1967; 87: 59-83.

11 Theodore FH. Points in the treatment of bacterial corneal ulcers. In: Leopold IH, ed. Symposium on ocular therapy. St Louis: Mosby, 1972; 5: 48-54.

12 Burns RP. Pseudomonas aeruginosa keratitis: mixed infections of the eye. Am $\mathcal{O}$ Ophthalmol 1969; 67: 257-62.

13 Wilson LA. Acute bacterial infection of the eye: bacterial keratitis and endophthalmitis. Trans Ophthalmol Soc UK 1986; 105: 43-60.

14 Di Bisceglie AM, Carmichael TR. Factors predisposing to central corneal ulceration in a developing population. $S$ Afr Med F 1987; 71: 769-70. 